\title{
Selenium Deficiency Is Associated with Mortality Risk from COVID-19
}

\author{
Arash Moghaddam ${ }^{1}$, Raban Arved Heller 2,3 ${ }^{\mathbb{D}}$, Qian Sun ${ }^{3}$, Julian Seelig ${ }^{3}$ (D), Asan Cherkezov ${ }^{1}$, \\ Linda Seibert ${ }^{1}$, Julian Hackler ${ }^{3}$, Petra Seemann ${ }^{3}$, Joachim Diegmann ${ }^{1}$, Maximilian Pilz ${ }^{4}$ (D), \\ Manuel Bachmann ${ }^{1}$, Waldemar B. Minich ${ }^{3}$ and Lutz Schomburg ${ }^{3, *(D)}$
}

1 ATORG, Aschaffenburg Trauma and Orthopedic Research Group, Center for Orthopedics, Trauma Surgery and Sports Medicine, Hospital Aschaffenburg-Alzenau, D-63739 Aschaffenburg, Germany; arash.moghaddam-alvandi@klinikum-ab-alz.de (A.M.); asan.cherkezov@stud-mail.uni-wuerzburg.de (A.C.); linda-glaab@t-online.de (L.S.); Joachim.Diegmann@klinikum-ab-alz.de (J.D.);

manuel.bachmann.md@gmail.com (M.B.)

2 HTRG, Heidelberg Trauma Research Group, Center for Orthopedics, Trauma Surgery and Spinal Cord Injury, Heidelberg University Hospital, D-69118 Heidelberg, Germany; raban.heller@charite.de

3 Institute for Experimental Endocrinology, Charité-Universitätsmedizin Berlin, Corporate Member of Freie Universität Berlin, Humboldt-Universität zu Berlin, and Berlin Institute of Health, D-13353 Berlin, Germany; qian.sun@charite.de (Q.S.); julian.seelig@charite.de (J.S.); julian.hackler@charite.de (J.H.); petra.seemann@charite.de (P.S.); waldemar.minich@charite.de (W.B.M.)

4 Institute of Medical Biometry and Informatics, Heidelberg University Hospital, Im Neuenheimer Feld 130.3, D-69120 Heidelberg, Germany; pilz@imbi.uni-heidelberg.de

* Correspondence: lutz.schomburg@charite.de; Tel.: +49-30-450524289; Fax: +49-30-450524922

Received: 5 July 2020; Accepted: 12 July 2020; Published: 16 July 2020

check for updates

\begin{abstract}
SARS-CoV-2 infections underlie the current coronavirus disease (COVID-19) pandemic and are causative for a high death toll particularly among elderly subjects and those with comorbidities. Selenium (Se) is an essential trace element of high importance for human health and particularly for a well-balanced immune response. The mortality risk from a severe disease like sepsis or polytrauma is inversely related to Se status. We hypothesized that this relation also applies to COVID-19. Serum samples $(n=166)$ from COVID-19 patients $(n=33)$ were collected consecutively and analyzed for total Se by X-ray fluorescence and selenoprotein P (SELENOP) by a validated ELISA. Both biomarkers showed the expected strong correlation $(r=0.7758, p<0.001)$, pointing to an insufficient Se availability for optimal selenoprotein expression. In comparison with reference data from a European cross-sectional analysis (EPIC, $n=1915)$, the patients showed a pronounced deficit in total serum Se (mean \pm SD, $50.8 \pm 15.7$ vs. $84.4 \pm 23.4 \mu \mathrm{g} / \mathrm{L})$ and SELENOP $(3.0 \pm 1.4 \mathrm{vs} .4 .3 \pm 1.0 \mathrm{mg} / \mathrm{L})$ concentrations. A Se status below the 2.5 th percentile of the reference population, i.e., [Se] $<45.7 \mu \mathrm{g} / \mathrm{L}$ and [SELENOP] $<2.56 \mathrm{mg} / \mathrm{L}$, was present in $43.4 \%$ and $39.2 \%$ of COVID samples, respectively. The Se status was significantly higher in samples from surviving COVID patients as compared with non-survivors (Se; $53.3 \pm 16.2$ vs. $40.8 \pm 8.1 \mu \mathrm{g} / \mathrm{L}$, SELENOP; $3.3 \pm 1.3$ vs. $2.1 \pm 0.9 \mathrm{mg} / \mathrm{L}$ ), recovering with time in survivors while remaining low or even declining in non-survivors. We conclude that Se status analysis in COVID patients provides diagnostic information. However, causality remains unknown due to the observational nature of this study. Nevertheless, the findings strengthen the notion of a relevant role of Se for COVID convalescence and support the discussion on adjuvant Se supplementation in severely diseased and Se-deficient patients.
\end{abstract}

Keywords: trace element; inflammation; selenoprotein P; micronutrient; COVID-19 


\section{Introduction}

Severe acute respiratory syndrome coronavirus-2 (SARS-CoV-2) infections underlie the current coronavirus disease (COVID-19) pandemic and are causative for an increasingly high death toll particularly among elderly subjects and those who have severe comorbidities, e.g., chronic obstructive pulmonary disease, hypertension, diabetes, cancer, or a combination thereof $[1,2]$. It has been reported that severe disease course often associates with an overreaction of the body's immune system with a massive cytokine and chemokine release ("cytokine storm") [3]. Accordingly, the attempts at controlling the inflammation by immunosuppressive treatment using, e.g., high dosages of corticosteroids have shown promising effects in reducing the rate of fatal disease course among the severely diseased COVID patients on mechanical ventilation (medRxiv 2020.06.22.20137273), causing a surge in dexamethasone demand [4]. This treatment success is reminiscent of the positive reports on dexamethasone being capable of positively affecting the course of severe acute respiratory distress syndrome [5], or of reducing the mortality rate in severely diseased and delirious patients from typhoid fever [6]. The strategy of repurposing common drugs known to positively affect the immune response are now increasingly applied in the current COVID pandemic [7]. The positive effects with tocilizumab and sarilumab are the most recent examples (NCT04306705, NCT04322773). An adjuvant supply of certain micronutrients as positive modulators of the immune system may further support these attempts, and some vitamins (A, B6, B12, C, D, and E) and essential trace elements (zinc, iron, selenium (Se), magnesium, or copper) are discussed as particularly promising [8]. However, at present, the data base is very small in relation to these micronutrients, and it is unknown whether certain vitamins or trace elements are indeed deficient in patients with COVID-19, and whether the concentrations are related to disease severity or mortality risk.

For several reasons, the essential trace element Se is of particular relevance for viral infections among these nutritional factors. The immune system relies on a set of specific selenoproteins containing selenocysteine in their active sites and known to depend on abundant Se supply for their full expression and enzymatic activities $[9,10]$. Se deficiency is an established risk factor for viral infections [11]. Pathogens show higher mutation rates in Se-deficient subjects and can decisively contribute to a rapid evolution of pathogenic viral species [12]. Keshan disease is an endemic cardiomyopathy related to Se deficiency, and supplemental Se has proven meaningful for reducing the virus-associated disease incidence [13]. Se deficiency is also a risk factor for death from severe disease, as shown, e.g., in sepsis [14] or polytraumatic injury [15]. Notably, the cure rate from COVID-19 was recently associated with basal Se status in different areas of China [16]. Collectively, the available studies support the notion that Se may be of relevance for infection with SARS-CoV-2 and disease course of COVID-19 [17-19]. However, data on Se status of individual patients severely affected by COVID-19 are missing. We hypothesized that severe Se deficiency is prevalent among the patients and associates with poor survival odds in COVID-19.

\section{Materials and Methods}

\subsection{Study Design}

A cross-sectional study of patients with COVID-19 was conducted at the non-profit Public Hospital Klinikum Aschaffenburg-Alzenau, Germany. Diagnosis of COVID-19 was based on positive detection of viral RNA using RT-PCR (real-time PCR-E-Gen according to Corman et al. [20], Medizinisches Versorgungszentrum MVZ Labor PD Dr. Volkmann \& Kollegen GbR, Karlsruhe, Germany). The study was conducted in accordance with the Declaration of Helsinki. Ethical counselling was provided by the authorities in Bavaria, Germany (Ethik-Kommission der Bayerischen Landesärztekammer, EA No. \#20033), and the study was registered at the German Clinical Trial Register (Deutsches Register Klinischer Studien, ID: DRKS00022294). All patients enrolled into the analysis or next of kin provided written informed consent. The number of blood drawings per patient was [median (IQR)] 4 (4) or [mean \pm SD] $5.03 \pm 4.27$ samples/patient. The samples were stored at $-80^{\circ} \mathrm{C}$ (Aschaffenburg, Germany) and sent on 
dry ice to a remote lab from the clinics for analysis (Charité Universitätsmedizin Berlin, Berlin, Germany). All measurements were conducted by scientists and technicians blinded to the clinical information. Reference values were derived from a comprehensive dataset of adult subjects participating in the European Prospective Investigation into Cancer and Nutrition (EPIC) study, analyzed by the same technology as published recently [21].

\subsection{Trace Element Analysis}

Total reflection X-ray fluorescence (TXRF) was used to determine the concentration of Se in serum samples using a benchtop TXRF spectrometer (S4 T-STAR, Bruker Nano GmbH, Berlin, Germany). Briefly, samples were diluted with a gallium standard, applied to polished quartz glass slides and dried overnight. Seronorm serum standard (Sero AS, Billingstad, Norway) served as control. The concentrations measured were within the specified range of the standard, and the inter-assay coefficient of variation (CV) was below $5 \%$ at a concentration of $45 \mu \mathrm{g}$ Se/L serum.

\subsection{SELENOP Quantification by ELISA}

SELENOP concentrations were measured from the serum samples by a sandwich method with monoclonal antibodies against human SELENOP using a validated commercial SELENOP-specific ELISA (selenOtest ELISA, selenOmed GmbH, Berlin, Germany) as described [22]. Quality of measurements was verified by including two human serum standards in each assay run. The inter-assay CV was below $15 \%$ during the analyses.

\subsection{Assessment of Glutathione Peroxidase-3 (GPx3) Activity}

The activity of glutathione peroxidase-3 (GPx3) was assessed by a coupled enzymatic test procedure monitoring nicotinamide adenine dinucleotide phosphate (NADPH) consumption at $340 \mathrm{~nm}$, as described earlier $[23,24]$. Briefly, serum samples were incubated with enzyme buffer containing $3.4 \mathrm{mM}$ reduced

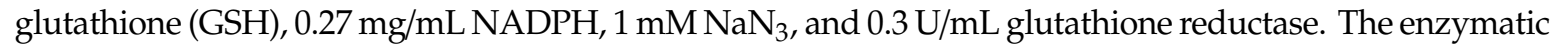
reaction was started by hydrogen peroxide, and consumption of NADPH was monitored at $340 \mathrm{~nm}$. Inter- and intra-assay CVs were below 20\%.

\subsection{Statistical Analysis}

Statistical analysis was performed with GraphPad Prism (Version 7, GraphPad Software Inc., San Diego, CA, USA) and the open software R, version 3.6.0 [25], applying the packages "tidyr" [26], "dplyr" [27], "pROC" [28], and "ggplot2" [29]. The Shapiro-Wilk test was used for assessing the normal distribution of values. Categorical variables were evaluated by Boschloo's test [30]. Comparisons were conducted by unpaired Student's t-test. More than two groups were compared with ANOVA and Dunn's multiple comparisons test. Correlations were tested by Spearman's correlation test. Differences between ROC curves were assessed by applying the DeLong's test for two correlated ROC curves. All statistical tests were two-sided, and $p$-values $<0.05$ were considered significant; ${ }^{*} p<0.05,{ }^{* *} p<0.01$, *** $p<0.001$, and ${ }^{* * * *} p<0.0001$.

\section{Results}

\subsection{Patient Characteristics}

A total of $n=33$ patients qualified for analysis and were enrolled into this observational study, providing a set of $n=166$ consecutive serum samples. COVID-19 patients who survived or died showed similar characteristics, except for a lower age range of the survivors (Table 1). 
Table 1. Characteristics of the COVID-19 patients contributing to this study.

\begin{tabular}{cccc}
\hline & Death & Discharge & Total \\
\hline $\begin{array}{c}\text { Sex } \\
\text { female } \\
\text { male }\end{array}$ & $4(67 \%)$ & $15(56 \%)$ & $19(58 \%)$ \\
Age & $2(33 \%)$ & $12(44 \%)$ & $14(42 \%)$ \\
median (IQR) & & & \\
Comorbidities & $89(81,94)$ & $69(38,91)$ & $77(38,94)$ \\
hypertension & & & \\
diabetes & $4(67 \%)$ & $18(67 \%)$ & $22(67 \%)$ \\
COPD & $2(33 \%)$ & $4(15 \%)$ & $6(18 \%)$ \\
CVD & $0(0 \%)$ & $1(4 \%)$ & $1(3 \%)$ \\
cerebrovascular disease & $3(50 \%)$ & $14(52 \%)$ & $17(52 \%)$ \\
adipositas & $1(17 \%)$ & $5(19 \%)$ & $6(18 \%)$ \\
Time to discharge or death * [d] & $1(17 \%)$ & $6(22 \%)$ & $7(21 \%)$ \\
median (IQR) & $10(2,32)$ & $19(3,46)$ & $15(2,46)$ \\
\hline
\end{tabular}

* Death in combination with COVID-19 diagnosis, irrespective of final mortality cause.

\subsection{Selenium (Se) Status Analysis}

Serum Se status was evaluated from all patient samples as assessed by three complementary biomarkers, i.e., total serum Se and SELENOP concentrations, as well as GPx3 activity. The three Se status biomarkers showed significant and linear correlations over the full range of data, indicating a high quality of the samples (Figure 1). The correlation coefficients were highest for the parameter pair of total serum Se and SELENOP concentration (Figure 1A), followed by the parameter pair GPx3 activity and total serum Se (Figure 1B). GPx3 activity and serum SELENOP concentration showed the least stringent correlation (Figure 1C).
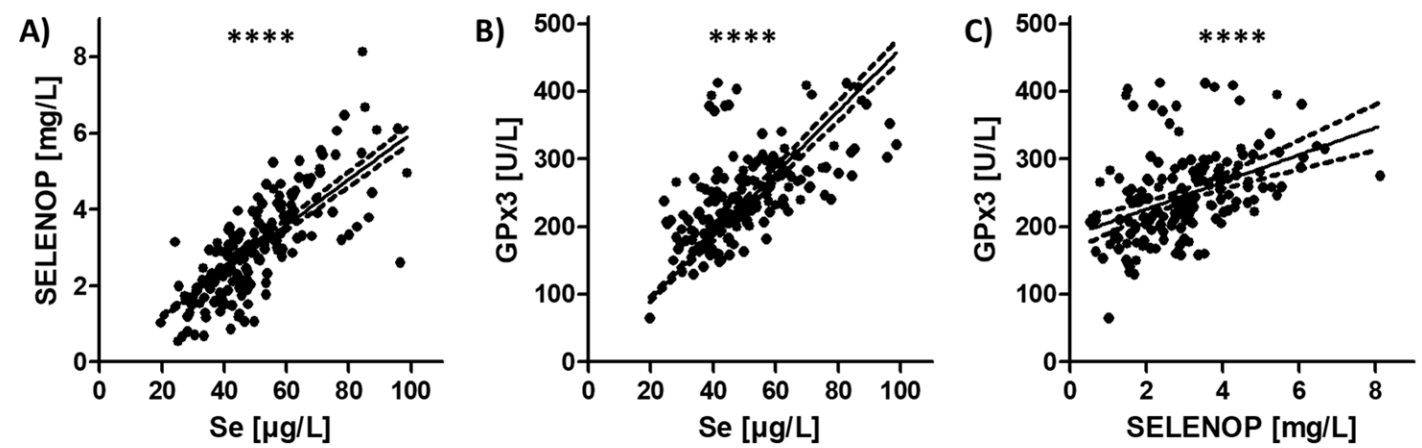

Figure 1. Analysis of Se status from samples of patients suffering from COVID-19 by three complementary serum biomarkers. Serum samples $(n=166)$ were analyzed from COVID-19 patients ( $n=33$ ) by measuring total Se concentration, serum SELENOP level, and activity of secreted GPx3.

(A) The Se transporter SELENOP and total Se concentration showed a tight positive linear correlation $(r=0.7896)$, in agreement with the analysis of (B) GPx3 activity and total Se concentration $(r=0.6239)$, as well as with (C) GPx3 activity and SELENOP concentration $(r=0.4954) . r$ : Spearman correlation coefficient (2-sided, 2-tailed), ${ }^{* * * *} p<0.0001$.

\subsection{Se Status of COVID-19 Patients in Relation to Reference Range of Healthy Control Subjects}

An average population-wide Se status was deduced from $n=1915$ datasets obtained earlier from healthy adult subjects participating in the cross-sectional EPIC study [21]. Reference ranges for total serum Se and SELENOP concentrations were deduced by determination of the 2.5th-97.5th percentile of the data. According to this large cross-sectional study, SELENOP concentrations are unrelated to age [21]. The chosen criterion of $95 \%$ of data constituting the reference ranges classifies a normal Se status when residing in the range of $45.7-131.6 \mu \mathrm{g} / \mathrm{L}$ for serum Se, and $2.56-6.63 \mathrm{mg} / \mathrm{L}$ for serum 
SELENOP concentration, respectively. According to these reference ranges, $44.4 \%$ of samples from COVID-19 patients were deficient in Se, and 39.6\% were deficient in SELENOP.

\subsection{Se Status of COVID-19 Patients in Relation to Survival}

When separating patient samples from surviving vs. deceased COVID-19 patients, the difference becomes more obvious. In the samples of deceased COVID-19 patients, $64.7 \%$ and $70.6 \%$ showed Se and SELENOP deficiency, respectively, whereas $39.3 \%$ and $32.6 \%$ of the samples from the survivors had to be classified as Se- and SELENOP-deficient, respectively. Accordingly, a significantly lower Se status was identified in the non-survivors in comparison with the survivors with respect to all three biomarkers of Se status analyzed (Table 2).

Table 2. Comparison of Se status biomarkers in COVID-19 samples in relation to survival.

\begin{tabular}{ccccc}
\hline & All Samples & Discharge & Death & $p$-Value * \\
\hline serum Se $[\mu \mathrm{g} / \mathrm{L}]$ & $n=166$ & $n=132$ & $n=34$ & $p<0.001$ \\
serum SELENOP [mg/L] & $50.8 \pm 15.7$ & $\mathbf{5 3 . 3} \pm \mathbf{1 6 . 2}$ & $40.8 \pm 8.1$ & \\
serum GPx3 [U/L] & $3.0 \pm 1.4$ & $3.3 \pm 1.3$ & $\mathbf{2 . 1} \pm \mathbf{0 . 9}$ & $p<0.001$ \\
& $246.1 \pm 64.4$ & $\mathbf{2 5 1 . 6 \pm 6 9 . 6}$ & $\mathbf{2 2 4 . 8} \pm 30.3$ & $p<\mathbf{0 . 0 0 1}$ \\
\hline
\end{tabular}

* Student's $t$-test, 2-tailed, 2-sided, comparison of discharge vs. death (bold numbers).

A comparison of the median values and inter quartile ranges (IQR) of the samples from the COVID-19 patients who did not survive in relation to the reference cohort of healthy adult European subjects indicates that the groups differ strongly, i.e., the IQR do not overlap. This means that the ranges encompassing $75 \%$ of all samples are separated from each other, irrespective of the biomarker used, i.e., both in relation to total serum Se and serum SELENOP concentrations (Figure 2A,B). Notably, the bottom $75 \%$ of values from the deceased patients are below the median values of the surviving COVID-19 patients, suggesting that both parameters of Se status are of value for the identification of patients with severe disease course and high mortality risk.

With regard to the choice of biomarker, both total serum Se and SELENOP concentrations appear similarly suitable for providing information on survival chances of COVID-19 patients. Importantly, Se and SELENOP showed the known positive linear correlation in both the group of non-survivors and of the surviving patients that were successfully discharged (Figure 2C,D).

The samples available for analysis were from different points in time as leftover serum from routine laboratory analyses. Hereby, it was possible to conduct a time-resolved analysis of changes in Se status of surviving vs. deceased COVID-19 patients. The analysis highlights that the Se status in patients surviving the disease tended to recover from the low values observed at admittance to the hospital, whereas no such positive development was observed in the non-survivors (Figure 3). 

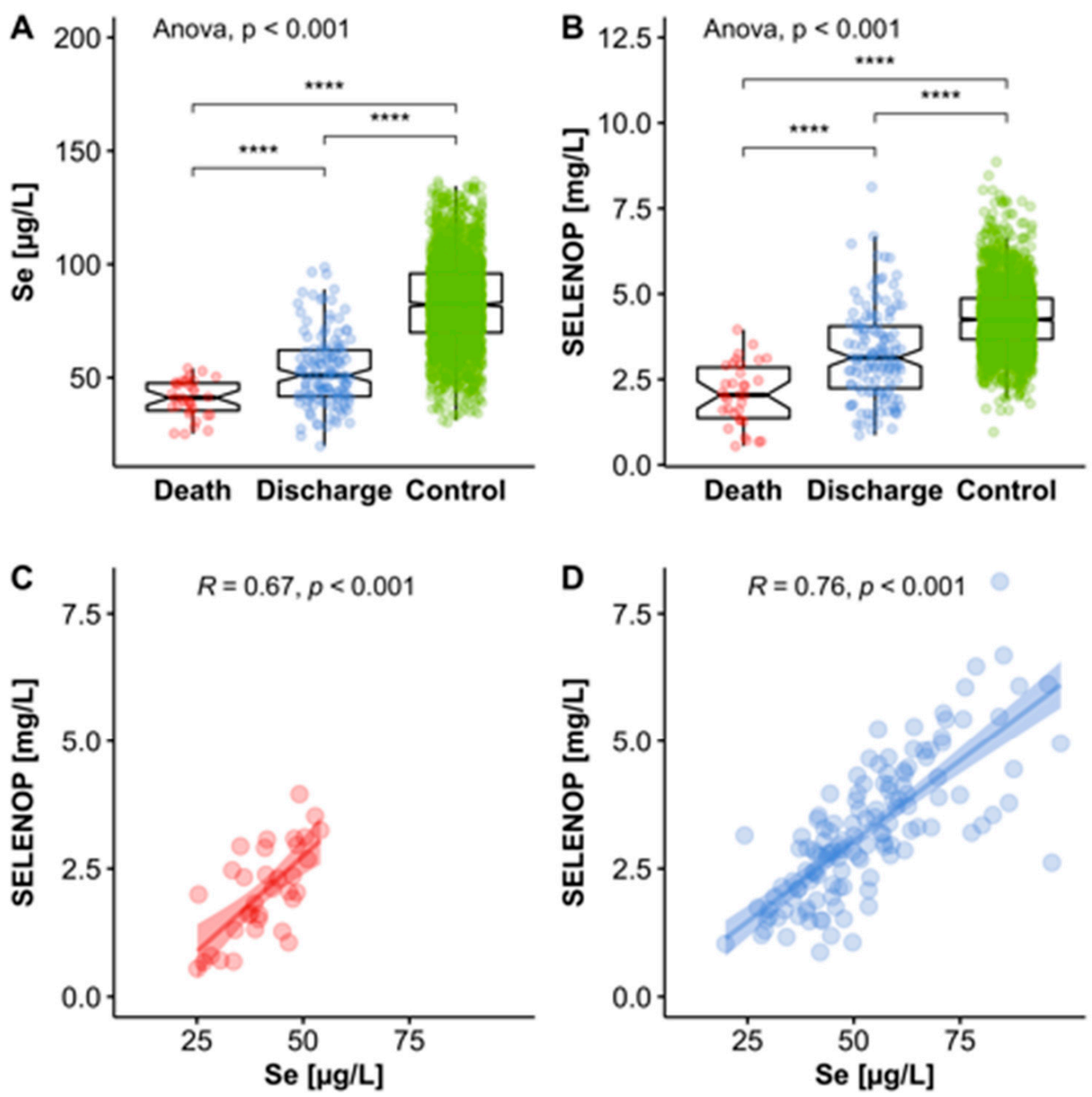

Figure 2. Comparison of Se status in COVID-19 patients who survived or died in relation to healthy controls. (A) Total serum Se concentrations differed significantly and were most strongly depressed in COVID-19 patients who did not survive. (B) SELENOP concentrations differed to a similar extent and were also lowest in non-survivors. (C) As observed in the full cohort of samples, Se and SELENOP showed a strong positive correlation in the group of non-survivors, as well as (D) in the group of survivors, albeit across a smaller and more limited concentration range in the non-survivors. All tests were two-sided and $p$-values $<0.05$ were considered statistically significant; $R$ : Spearman correlation coefficient (2-sided, 2-tailed), ${ }^{* * * *}$ indicates $p<0.0001$.

A direct comparison of Se status in COVID-19 patients to reference values for the activity of GPx3 as a biomarker was not possible, as GPx3 had not been determined in the samples of the large reference cohort from the EPIC study [21]. 


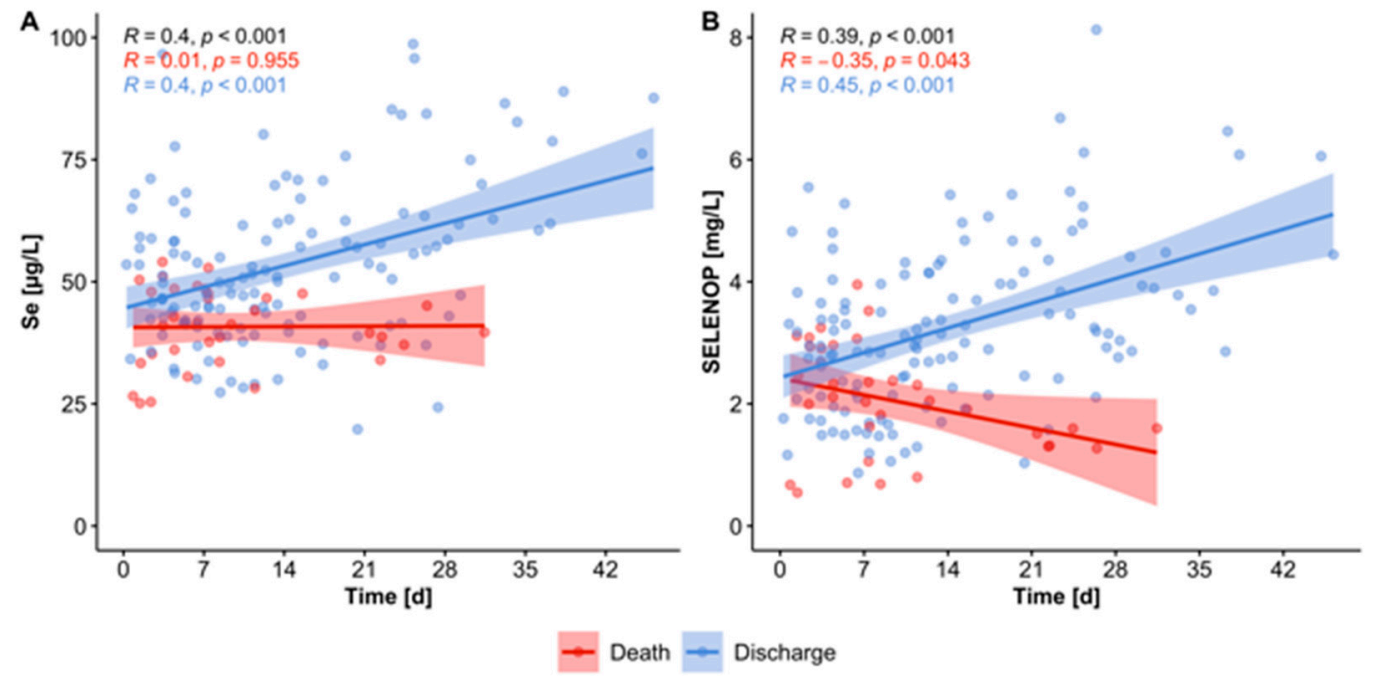

Figure 3. Time-resolved changes in Se status in surviving vs. deceased patients. Serum samples from COVID-19 patients were analyzed for (A) total Se, and (B) serum SELENOP concentrations. Surviving patients (blue dots) showed increasing Se status with time, with respect to both serum Se and SELENOP. In comparison, Se status remained constant, and SELENOP concentrations declined, respectively, in non-surviving patients (red dots). All tests were two-sided and $p$-values $<0.05$ were considered statistically significant; R: Spearman correlation coefficient (2-sided, 2-tailed); overall, deathand discharge-related correlations of Se status vs. time are indicated in the upper left corners.

Next, a receiver operating characteristic (ROC) curve analysis was conducted to analyze the diagnostic ability of the Se status biomarkers for survival odds. ROC curve analyses can contribute to decision-making in a binary classifier system by testing a discrimination threshold via calculating all possible variations. However, ROC plots alone may be misleading and bear the risk of error when applied in imbalanced classification scenarios [31]. For this reason, a precision recall curve (PRC) was calculated to identify the fraction of true positives among all the positive predictions, thereby providing a more accurate prediction of future classification performance (Figure 4). The available data on SELENOP, Se, and GPx3 were suitable to reliably distinguish between those patients who could be discharged and those who died, respectively. Applying a stepwise Akaike information criteria (AIC) selection process revealed that the SELENOP concentration outperformed the other variables as well as combinations thereof. This result is mirrored in both the corresponding ROC and PRC curves (Figure 4A,B). Calculating the area under the curve (AUC) for the three Se status biomarkers indicates a better suitability of total serum Se and SELENOP concentrations in comparison with GPx3 activity for diagnosis and prediction, i.e., $75.9 \%$ for SELENOP and $74.2 \%$ for total Se, respectively, vs. $62.4 \%$ for GPx3 activity.

As it is known that age strongly predisposes to severe disease course and mortality risk in COVID-19, an analysis for serum Se and SELENOP in combination with age was conducted. The model based on SELENOP was slightly superior to the model based on Se. Significant differences were detected between the models based on either SELENOP or Se and GPx3 (DeLong's test; AUC (SELENOP) $=75.9 \%$ vs. $\mathrm{AUC}(\mathrm{GPx} 3)=62.4 \%, p=0.004 ; \mathrm{AUC}(\mathrm{Se})=74.2 \%$ vs. $\mathrm{AUC}(\mathrm{GPx} 3)=62.4 \%, p=0.007)$. The final univariate model based on SELENOP yielded an AUC of 75.9\%. The optimal cutpoint based on Youden's J statistic was chosen at $3.1 \mathrm{mg} / \mathrm{L}$ (Figure 4A). This cutpoint is characterized by a sensitivity of $91.2 \%$ and a specificity of $50.8 \%$, and may serve as a valuable screening tool to contribute to a better assessment of the mortality risk in patients suffering from COVID-19. This is also reflected in the precision recall curve (PRC) recommended in such analyses [31] (Figure 4B). In view of the limited sample size of $n=33$ patients only, the Se status biomarkers were analyzed in a univariate modeling process via stepwise backwards AIC selection to avoid an overfit. The favored SELENOP-based model was finally adjusted for the patients' age and resulted in an increased AUC of 94.8\% (Figure 4C). 

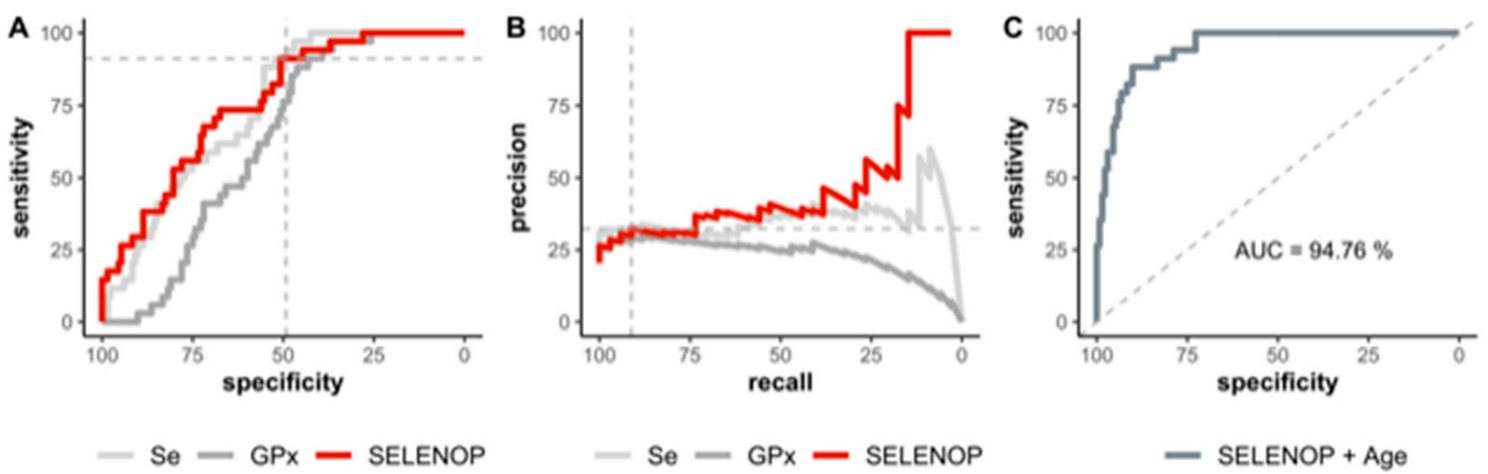

Figure 4. Receiver operating characteristics (ROC) analyses of Se status biomarkers in relation to risk of death from COVID-19. (A) ROC analyses as univariate prediction models based on the serum concentrations of SELENOP, Se, and GPx3 (pooled values from admission to the endpoint of the study) are capable of discriminating between patients that died and those that have been discharged. The optimal cutpoint of SELENOP concentrations at $3.1 \mathrm{mg} / \mathrm{L}$ according to Youden's J statistics is indicated by the point where the dashed grey lines cross. (B) The corresponding precision recall curve (PRC) indicates the fraction of true positives among all the positive predictions and may serve as a meaningful addition to current risk estimates. The corresponding cutpoint is again indicated. (C) ROC analysis of SELENOP status in relation to risk of death from COVID-19 with respect to the patients' age. The area under the curve (AUC) is indicated below the diagonal 50\% line.

This notion is further underlined by the specific characteristics of the predictive models used (Table 3).

Table 3. Specific characteristics of the predictive models used. For each model, the variable estimates included in the calculations are provided with their corresponding confidence interval (CI).

\begin{tabular}{ccccc}
\hline & Serum Se & Serum SELENOP & GPx3 Activity & All \\
\hline (intercept) & $-1.70^{* * *}[-2.20,-1.20]$ & $-1.75^{* * *}[-2.27,-1.24]$ & $-1.42^{* * *}[-1.81,-1.02]$ & $-1.80^{* * *}[-2.34,-1.26]$ \\
\hline Se & $-1.19^{* * *}[-1.79,-0.60]$ & & & $-0.55[-1.39,0.30]$ \\
\hline SELENOP & & $-1.28^{* * *}[-1.86,-0.70]$ & & $-0.94{ }^{*}[-1.72,-0.16]$ \\
\hline GPx3 & & & $-0.46^{*}[-0.89,-0.04]$ & $0.09[-0.37,0.54]$ \\
\hline $\mathbf{N}$ & 166 & 166 & 166 & 166 \\
\hline AIC & 150.5 & 146.3 & 167.3 & 148.5 \\
\hline Pseudo R & 0.19 & 0.23 & 0.05 & 0.24 \\
\hline \multicolumn{2}{r}{ All continuous predictors are mean-centered and scaled by 1 standard deviation. ${ }^{* * *} p<0.001 ; *$} & $p<0.05$.
\end{tabular}

\section{Discussion}

In this manuscript, we report that patients suffering from COVID-19 display a deficiency in the essential trace element Se in blood, along with low concentrations of the Se transporter SELENOP and low enzymatic activity of the secreted GPx3. Notably, the Se deficiency was very strong in comparison with healthy European adults, and it was reflected concordantly in relatively depressed readings of all three different Se status biomarkers determined. The observation that Se deficiency was more severe in the samples obtained from non-survivors as compared with survivors of COVID-19 may suggest some relevance of the trace element for coping with the virus and for successful convalescence. This hypothesis is also supported by the difference in Se status development in time, with survivors displaying a progressively recovering Se status, while the non-survivors do not.

Besides the physiological role of Se for supporting the biosynthesis of immune system-relevant selenoproteins, the data also highlight that a determination of Se status by any of the biomarkers evaluated is of diagnostic value for a better prediction of disease course and an improved identification of patients at particular risk for losing the battle against this devastating infection. However, reliable 
Se analysis is not readily available at all clinics and hospitals, and many commercial or experimental analytical test systems for SELENOP quantification are not yielding accurate results [32], causing confusion and disarray [33]. For this reason, the issue of avoiding severe Se deficiency in the preventive and clinical settings by using a respectively balanced diet or suitable supplements may be the most urgent and meaningful consequence from the interaction between Se deficiency and mortality risk observed in this study.

Although the nature of the analysis as an observational study does not allow the deduction of causal relationships, there are different hypotheses for the underlying biochemical pathways leading to the observations presented in this manuscript.

Firstly, Se status may already have been relatively low in the patients before disease, constituting a risk factor for viral infection as shown previously for other diseases [11,12]. In this respect, the experiences with viral-induced Keshan disease [13] or AIDS [34] may serve as paradigmatic examples highlighting the potential relevance of Se for infection risk and disease course [34]. However, the high infection rate of SARS-CoV-2 apparently infecting very many of the directly exposed subjects [35] in combination with the majority of COVID-19 samples exhibiting Se values below the 2.5 th percentile of the population range argues against a Se-dependent predisposition as an explanation for the findings.

Secondly, in disease and upon the growing inflammation, a potentially pre-existing low Se status may decline further. This notion is supported from similar findings in other severe diseases, especially sepsis [14] and polytraumatic injury [15], where low, declining, and mortality-relevant Se deficiency has been observed that is unlikely a predisposition. Moreover, the negative acute phase response of hepatic SELENOP biosynthesis [36], together with the suppressive effects of hypoxia [37] or cytokines, e.g., IL-6 [38], argue in favor of this mechanism contributing to the differences.

Thirdly, a longer stay on the ICU under inflammatory and hypoxic conditions may cause an elevated Se requirement due to ongoing Se loss, as erythrocyte Se often remains normal despite declining Se in blood [39]. In human evolution, high-quality medical care with supportive ventilation was usually not available, and an infection was followed soon by either remission or death. Under these conditions, safeguarding essential micronutrients for later recovery was no survival advantage. The present care on the ICU over long periods of time constitutes a fundamental different situation, where the constant suppression of hepatic SELENOP biosynthesis may require supplemental measures in the long run [39]. Concordant with this notion, the hypothesized association of low Se status with impaired recovery was reported from an in silico analysis of cure rates from COVID-19 in the different areas of China with diverging baseline Se status [16].

Fourthly, an over-shooting immune response may be directly related to Se status as oxidative stress may overrun the capacity of protective selenoenzymes of the GPx and thioredoxin reductase families and low molecular weight antioxidants [40]. This loss of redox balance has been hypothesized before as of potential etiopathogenic relevance [12,41]. The therapeutic success of dexamethasone or tocilicumab treatment, as well as the perspective of the GPx mimetic ebselen as a promising therapeutic measure lend further support to this theory [42,43].

Finally, a declining serum Se status may just constitute a surrogate marker for disease severity and the tone of pathological stressors, like hypoxia and inflammatory cytokines. This notion is supported by a vast body of literature on declining selenoprotein biosynthesis under acute phase conditions, in inflammation and under hypoxia. A declining Se status will further disrupt the redox balance thereby closing a fatal feed-forward loop, again arguing for the potential relevance of some supplemental support to interrupt this vicious cycle during long-lasting disease (Figure 5).

Collectively, similar to the proposed interrelation of declining Se status in malignant diseases, the strong deficit in Se and SELENOP observed in COVID-19 may result from a combination of the aforementioned pathways and interactions. Supportive measures aimed at improving selenoprotein biosynthesis in COVID-19 may enable a better redox control and fine-tuned response of the immune system [41]. It appears meaningful, timely, and promising to initiate population-wide measures trying to identify subjects with pre-existing Se deficits, not just as a preventive measure for viral infections, 
spread, and virulence development [11,12,42], but also to reduce the individual risk for cardiovascular mortality [44-47], cancer [21,48,49], and death from severe disease [10,14,39].

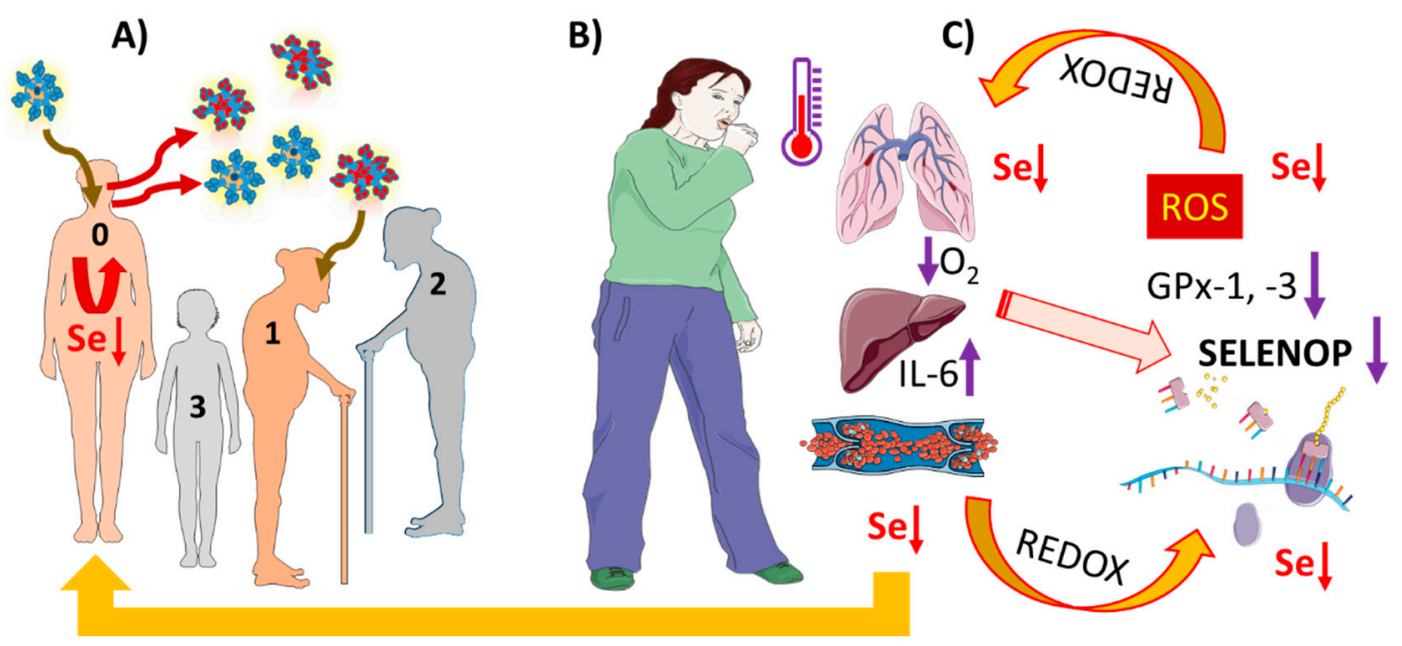

Figure 5. Pathophysiological mechanisms potentially underlying low Se status in severe COVID-19. Infections by SARS-CoV-2 occur largely independent from baseline Se status. (A) Some individuals with a poor immune system and low baseline Se status (0) may spread the virus (blue) efficiently and allow viral replication and rapid evolution of particular pathogenic viral species (red) due to low expression of protective selenoenzymes. Subjects with better Se status (1-3) may be less prone to severe disease course. (B) COVID-19 is characterized by inflammation, hypoxia, and high cytokine concentrations (e.g., IL-6). The combination of hypoxia and IL-6 suppresses selenoprotein expression. (C) Biosynthesis of the Se transporter SELENOP in hepatocytes is particularly sensitive, causing whole body Se status decline and insufficient expression of protective selenoenzymes, e.g., cytosolic GPx1 and plasma GPx3. Insufficient inactivation of peroxides as precursors of reactive oxygen species (ROS) results, causing a serious disturbance of redox balance, closing a vicious cycle both with respect to selenoprotein expression, Se concentrations, and COVID-19 progression. It is hypothesized that supplemental Se may interrupt this series of detrimental events and contribute to better odds for convalescence. This figure was created by using some Servier Medical Art templates, which are licensed under a Creative Commons Attribution 3.0 Unported License; https://smart.servier.com.

The particular strengths of the current study are the parallel assessment of different and coherent biomarkers of Se status by a standardized methodology, and the blinded set-up of the analyses. Among the limitations are, as usual in explorative pilot studies, the relatively limited number of patients and samples, and the lack of clinical data on inflammatory parameters.

\section{Conclusions}

COVID-19 constitutes a universal threat to human health, necessitating fast, promising, and safe measures for reducing infection risk, suppressing virulence development, strengthening the immune system, and supporting recovery. The essential trace element Se may be most relevant for these issues. Subjects residing in areas with poor baseline Se supply or on restricted nutrition, and COVID patients with pre-existing comorbidities or long disease course are at particularly elevated risk for severe Se deficiency, and may profit from improving the Se supply by dietary or supplemental measures. The observed association of mortality risk with Se deficit and the likely underlying feed-forward mechanism argues for initiating intervention studies under the highest quality standards, in order not to miss a universally available, inexpensive, and safe preventive measure and adjuvant treatment option. 
Author Contributions: Conceptualization, A.M., R.A.H. and L.S. (Lutz Schomburg); methodology, R.A.H., Q.S., J.S., A.C., J.H., P.S. and W.B.M.; software, R.A.H., A.C., L.S. (Linda Seibert), M.P. and M.B.; validation, R.A.H., Q.S., J.S., A.C., L.S. (Linda Seibert), J.H. and P.S.; formal analysis, A.M., R.A.H., A.C., L.S. (Linda Seibert), J.D., M.P., M.B., W.B.M. and L.S. (Lutz Schomburg); resources, A.M., M.B. and L.S. (Lutz Schomburg); data curation, R.A.H., Q.S., J.S., M.P. and L.S. (Lutz Schomburg); writing—original draft preparation, A.M., R.A.H. and L.S. (Lutz Schomburg); writing-review and editing, A.M., R.A.H., Q.S., J.S., A.C., L.S. (Linda Seibert), J.H., P.S., J.D., M.P., M.B., W.B.M. and L.S. (Lutz Schomburg); software, R.A.H., A.C., L.S. (Linda Seibert), M.P. and M.B.; visualization, R.A.H., J.S., A.C., L.S. (Linda Seibert), and M.P.; supervision, A.M. and L.S. (Lutz Schomburg); funding acquisition A.M. and L.S. (Lutz Schomburg). All authors have read and agreed to the published version of the manuscript.

Funding: The research has been funded by the Deutsche Forschungsgemeinschaft (DFG), Research Unit FOR-2558 "TraceAge" (Scho 849/6-1). We acknowledge financial support by the Open Access Publication Fund of Charité-Universitätsmedizin Berlin, and the funding received towards the doctoral thesis of $\mathrm{RH}$ from the Oskar-Helene-Heim foundation, Berlin, Germany.

Acknowledgments: We thank Vartitér Seher, Gabriele Boehm and Anja Fischbach for excellent technical support, Volker Daniel, University Hospital Heidelberg, Bahram Biglari, BG Trauma Centre Ludwigshafen, and Helena L. Crowell, University of Zurich, for constructive discussions, and two helpful and fast anonymous reviewers for excellent advice. Intellectual support and essential motivation was provided by valuable and inspiring colleagues from the International Society for Selenium Research (ISSR).

Conflicts of Interest: L.S. holds shares and P.S. serves as CEO of selenOmed GmbH, a company involved in Se status assessment and supplementation. The other authors declare no competing interest.

\section{References}

1. Guan, W.J.; Liang, W.H.; Zhao, Y.; Liang, H.R.; Chen, Z.S.; Li, Y.M.; Liu, X.Q.; Chen, R.C.; Tang, C.L.; Wang, T.; et al. Comorbidity and its impact on 1590 patients with covid-19 in china: A nationwide analysis. Eur. Respir. J. 2020, 55, 2000547. [CrossRef] [PubMed]

2. Wang, B.L.; Li, R.B.; Lu, Z.; Huang, Y. Does comorbidity increase the risk of patients with COVID-19: Evidence from meta-analysis. Aging 2020, 12, 6049-6057. [CrossRef]

3. Song, P.; Li, W.; Xie, J.; Hou, Y.; You, C. Cytokine storm induced by sars-cov-2. Clin. Chim. Acta 2020, 509, 280-287. [CrossRef] [PubMed]

4. Mahase, E. COVID-19: Demand for dexamethasone surges as recovery trial publishes preprint. BMJ 2020, 369, m2512. [CrossRef] [PubMed]

5. Villar, J.; Ferrando, C.; Martinez, D.; Ambros, A.; Munoz, T.; Soler, J.A.; Aguilar, G.; Alba, F.; Gonzalez-Higueras, E.; Conesa, L.A.; et al. Dexamethasone treatment for the acute respiratory distress syndrome: A multicentre, randomised controlled trial. Lancet Respir. Med. 2020, 8, 267-276. [CrossRef]

6. Hoffman, S.L.; Punjabi, N.H.; Kumala, S.; Moechtar, M.A.; Pulungsih, S.P.; Rivai, A.R.; Rockhill, R.C.; Woodward, T.E.; Loedin, A.A. Reduction of mortality in chloramphenicol-treated severe typhoid fever by high-dose dexamethasone. N. Engl. J. Med. 1984, 310, 82-88. [CrossRef]

7. Joshi, S.; Joshi, M.; Degani, M.S. Tackling sars-cov-2: Proposed targets and repurposed drugs. Future Med. Chem. 2020. [CrossRef]

8. Calder, P.C.; Carr, A.C.; Gombart, A.F.; Eggersdorfer, M. Optimal nutritional status for a well-functioning immune system is an important factor to protect against viral infections. Nutrients 2020, 12, 1181. [CrossRef]

9. Hoffmann, P.R.; Berry, M.J. The influence of selenium on immune responses. Mol. Nutr. Food Res. 2008, 52, 1273-1280. [CrossRef]

10. Schomburg, L. The other view: The trace element selenium as a micronutrient in thyroid disease, diabetes, and beyond. Hormones 2020, 19, 15-24. [CrossRef] [PubMed]

11. Guillin, O.M.; Vindry, C.; Ohlmann, T.; Chavatte, L. Selenium, selenoproteins and viral infection. Nutrients 2019, 11, 2101. [CrossRef] [PubMed]

12. Beck, M.A.; Handy, J.; Levander, O.A. Host nutritional status: The neglected virulence factor. Trends Microbiol. 2004, 12, 417-423. [CrossRef]

13. Zhou, H.; Wang, T.; Li, Q.; Li, D. Prevention of keshan disease by selenium supplementation: A systematic review and meta-analysis. Biol. Trace Elem. Res. 2018, 186, 98-105. [CrossRef] [PubMed]

14. Forceville, X.; Vitoux, D.; Gauzit, R.; Combes, A.; Lahilaire, P.; Chappuis, P. Selenium, systemic immune response syndrome, sepsis, and outcome in critically ill patients. Crit. Care Med. 1998, 26, 1536-1544. [CrossRef] [PubMed] 
15. Braunstein, M.; Kusmenkov, T.; Zuck, C.; Angstwurm, M.; Becker, N.P.; Bocker, W.; Schomburg, L.; Bogner-Flatz, V. Selenium and selenoprotein p deficiency correlates with complications and adverse outcome after major trauma. Shock 2020, 53, 63-70. [CrossRef]

16. Zhang, J.; Taylor, E.W.; Bennett, K.; Saad, R.; Rayman, M.P. Association between regional selenium status and reported outcome of covid-19 cases in china. Am. J. Clin. Nutr. 2020,111, 1297-1299. [CrossRef]

17. Fakhrolmobasheri, M.; Nasr-Esfahany, Z.; Khanahmad, H.; Zeinalian, M. Selenium supplementation can relieve the clinical complications of covid-19 and other similar viral infections. Int. J. Vitam. Nutr. Res. 2020, 9, 1-3. [CrossRef]

18. Kieliszek, M.; Lipinski, B. Selenium supplementation in the prevention of coronavirus infections (covid-19). Med. Hypotheses 2020, 143, 109878. [CrossRef]

19. Seale, L.A.; Torres, D.J.; Berry, M.J.; Pitts, M.W. A role for selenium-dependent gpx1 in sars-cov-2 virulence. Am. J. Clin. Nutr. 2020. [CrossRef]

20. Corman, V.M.; Landt, O.; Kaiser, M.; Molenkamp, R.; Meijer, A.; Chu, D.K.; Bleicker, T.; Brunink, S.; Schneider, J.; Schmidt, M.L.; et al. Detection of 2019 novel coronavirus (2019-ncov) by real-time rt-pcr. Eurosurveillance 2020, 25, 2000045. [CrossRef]

21. Hughes, D.J.; Fedirko, V.; Jenab, M.; Schomburg, L.; Meplan, C.; Freisling, H.; Bueno-de-Mesquita, H.B.; Hybsier, S.; Becker, N.P.; Czuban, M.; et al. Selenium status is associated with colorectal cancer risk in the european prospective investigation of cancer and nutrition cohort. Int. J. Cancer 2015, 136, 1149-1161. [CrossRef]

22. Hybsier, S.; Schulz, T.; Wu, Z.; Demuth, I.; Minich, W.B.; Renko, K.; Rijntjes, E.; Kohrle, J.; Strasburger, C.J.; Steinhagen-Thiessen, E.; et al. Sex-specific and inter-individual differences in biomarkers of selenium status identified by a calibrated elisa for selenoprotein p. Redox Biol. 2017, 11, 403-414. [CrossRef] [PubMed]

23. Schomburg, L.; Schweizer, U.; Holtmann, B.; Flohe, L.; Sendtner, M.; Kohrle, J. Gene disruption discloses role of selenoprotein p in selenium delivery to target tissues. Biochem. J. 2003, 370, 397-402. [CrossRef] [PubMed]

24. Mehl, S.; Sun, Q.; Gorlich, C.L.; Hackler, J.; Kopp, J.F.; Renko, K.; Mittag, J.; Schwerdtle, T.; Schomburg, L. Cross-sectional analysis of trace element status in thyroid disease. J. Trace Elem. Med. Biol. 2020, 58, 126430. [CrossRef]

25. R Core Team. R: A Language and Environment for Statistical Computing; The R Foundation for Statistical Computing: Vienna, Austria, 2020; Volume 1.

26. Wickham, H.; Henry, L. Tidyr: Tidy Messy Data; R Package Version 1.1.0; The R Foundation for Statistical Computing: Vienna, Austria, 2020; Available online: https://CRAN.R-project.org/package=tidyr (accessed on 14 July 2020).

27. Wickham, H.; Francois, R.; Henry, L.; Müller, K. Dplyr: A Grammar of Data Manipulation; R Package Version 1.0.0; The R Foundation for Statistical Computing: Vienna, Austria, 2020; Available online: https:/CRAN.R-project. org/package $=$ dplyr (accessed on 14 July 2020).

28. Robin, X.; Turck, N.; Hainard, A.; Tiberti, N.; Lisacek, F.; Sanchez, J.C.; Muller, M. Proc: An open-source package for $\mathrm{r}$ and $\mathrm{s}+$ to analyze and compare roc curves. BMC Bioinform. 2011, 12, 77. [CrossRef] [PubMed]

29. Wickham, H. Ggplot2: Elegant Graphics for Data Analysis; R Package Version 2.1.0; Springer: New York, NY, USA, 2009; pp. 1-195.

30. Boschloo, R.D. Raised conditional level of significance for $2 \times 2$ table when testing equality of 2 probabilities. Ann. Math. Stat. 1968, 39, 1094.

31. Saito, T.; Rehmsmeier, M. The precision-recall plot is more informative than the roc plot when evaluating binary classifiers on imbalanced datasets. PLoS ONE 2015, 10, e0118432. [CrossRef]

32. Saito, Y.; Misu, H.; Takayama, H.; Takashima, S.I.; Usui, S.; Takamura, M.; Kaneko, S.; Takamura, T.; Noguchi, N. Comparison of human selenoprotein p determinants in serum between our original methods and commercially available kits. Biol. Pharm. Bull. 2018, 41, 828-832. [CrossRef]

33. Schomburg, L.; Melander, O. Letter by schomburg and melander regarding article, "selenoprotein p promotes the development of pulmonary arterial hypertension: A possible novel therapeutic target". Circulation 2019, 139, 722-723. [CrossRef]

34. Harthill, M. Review: Micronutrient selenium deficiency influences evolution of some viral infectious diseases. Biol. Trace Elem. Res. 2011, 143, 1325-1336. [CrossRef] 
35. Wu, J.T.; Leung, K.; Bushman, M.; Kishore, N.; Niehus, R.; de Salazar, P.M.; Cowling, B.J.; Lipsitch, M.; Leung, G.M. Estimating clinical severity of covid-19 from the transmission dynamics in Wuhan, China. Nat. Med. 2020, 26, 506-510. [CrossRef] [PubMed]

36. Renko, K.; Hofmann, P.J.; Stoedter, M.; Hollenbach, B.; Behrends, T.; Kohrle, J.; Schweizer, U.; Schomburg, L. Down-regulation of the hepatic selenoprotein biosynthesis machinery impairs selenium metabolism during the acute phase response in mice. FASEB J. 2009, 23, 1758-1765. [CrossRef]

37. Becker, N.P.; Martitz, J.; Renko, K.; Stoedter, M.; Hybsier, S.; Cramer, T.; Schomburg, L. Hypoxia reduces and redirects selenoprotein biosynthesis. Metallomics 2014, 6, 1079-1086. [CrossRef] [PubMed]

38. Martitz, J.; Becker, N.P.; Renko, K.; Stoedter, M.; Hybsier, S.; Schomburg, L. Gene-specific regulation of hepatic selenoprotein expression by interleukin-6. Metallomics 2015, 7, 1515-1521. [CrossRef] [PubMed]

39. Manzanares, W.; Langlois, P.L.; Heyland, D.K. Pharmaconutrition with selenium in critically ill patients: What do we know? Nutr. Clin. Pract. 2015, 30, 34-43. [CrossRef] [PubMed]

40. Nordberg, J.; Arner, E.S. Reactive oxygen species, antioxidants, and the mammalian thioredoxin system. Free Radic. Biol. Med. 2001, 31, 1287-1312. [CrossRef]

41. Steinbrenner, H.; Al-Quraishy, S.; Dkhil, M.A.; Wunderlich, F.; Sies, H. Dietary selenium in adjuvant therapy of viral and bacterial infections. Adv. Nutr. 2015, 6, 73-82. [CrossRef]

42. Sies, H.; Parnham, M.J. Potential therapeutic use of ebselen for covid-19 and other respiratory viral infections. Free Radic. Biol. Med. 2020, 156, 107-112. [CrossRef]

43. Jin, Z.; Du, X.; Xu, Y.; Deng, Y.; Liu, M.; Zhao, Y.; Zhang, B.; Li, X.; Zhang, L.; Peng, C.; et al. Structure of $\mathrm{m}$ (pro) from sars-cov-2 and discovery of its inhibitors. Nature 2020, 582, 289-293. [CrossRef] [PubMed]

44. Alehagen, U.; Johansson, P.; Bjornstedt, M.; Rosen, A.; Post, C.; Aaseth, J. Relatively high mortality risk in elderly swedish subjects with low selenium status. Eur. J. Clin. Nutr. 2016, 70, 91-96. [CrossRef]

45. Schomburg, L.; Orho-Melander, M.; Struck, J.; Bergmann, A.; Melander, O. Selenoprotein-p deficiency predicts cardiovascular disease and death. Nutrients 2019, 11, 1852. [CrossRef] [PubMed]

46. Bomer, N.; Grote Beverborg, N.; Hoes, M.F.; Streng, K.W.; Vermeer, M.; Dokter, M.M.; Ijmker, J.; Anker, S.D.; Cleland, J.G.F.; Hillege, H.L.; et al. Selenium and outcome in heart failure. Eur. J. Heart Fail. 2019. [CrossRef]

47. Hu, X.F.; Stranges, S.; Chan, L.H.M. Circulating selenium concentration is inversely associated with the prevalence of stroke: Results from the canadian health measures survey and the national health and nutrition examination survey. J. Am. Heart Assoc. 2019, 8, e012290. [CrossRef] [PubMed]

48. Rayman, M.P. Selenium and human health. Lancet 2012, 379, 1256-1268. [CrossRef]

49. Schomburg, L.; Hughes, D.J. The missing link? The potential role of selenium in the development of liver cancer and significance for the general population. Expert Rev. Gastroenterol. Hepatol. 2017, 11, 707-709. [CrossRef] [PubMed]

(C) 2020 by the authors. Licensee MDPI, Basel, Switzerland. This article is an open access article distributed under the terms and conditions of the Creative Commons Attribution (CC BY) license (http://creativecommons.org/licenses/by/4.0/). 